\title{
A PATTERN META-MODEL FOR BUSINESS PROCESS IMPROVEMENT
}

\author{
Nesrine Missaoui ${ }^{1,3}$ and Sonia Ayachi Ghannouchi ${ }^{2,3}$ \\ ${ }^{1}$ University of Sousse, Higher Institute of Computer Science and Communication Techniques of Sousse ISITCOM, 4011, \\ Hammam Sousse, Tunisia \\ ${ }^{2}$ University of Sousse, Higher Institute of Management of Sousse, 4000, Sousse, Tunisia \\ ${ }^{3}$ University of Manouba, ENSI, RIADI Laboratory, 2010, Manouba, Tunisia
}

\begin{abstract}
Although there are many initiatives related to defining specific Business Process Improvement patterns (BPIP), we don't find any general description of such patterns. Determining the essential attributes constituting a BPIP can be very helpful in this context and will certainly contribute to describing the general structure of any improvement pattern. Thus, the purpose of this paper is to propose a meta-model, presenting a complete and global structure of an improvement pattern. The definition of the meta-model was based on a study of the different attributes defined in literature for existing meta-models, presenting not only the structure of a BPIP but also other types of patterns. The defined meta-model gives detailed information about the core attributes and types of BPIP while being based on the flexibility concept. The main benefit of this research study is presenting a complete and general meta-model for BPI patterns by defining the core attributes of a pattern as well those specific to a particular type of patterns, selected based on the context of application.
\end{abstract}

\section{KEYWORDS}

Business Process Improvement, Meta-Model, BPI Patterns, Business Process Flexibility

\section{INTRODUCTION}

Business process improvement (BPI) is an approach used to analyze, review and improve existing business processes by identifying inefficiencies and redesigning the process in order to reduce execution time and eliminate waste in a process. Indeed, the need for improving a business process (BP) has increased after the business process reengineering wave (Davenport, 1993) where it was adopted in many research works.

In fact, the main difference between BPR and BPI is that BPR presents revolutionary changes in a BP, while BPI focuses on the continuous improvement and the evolutionary changes of a process: it is considered as the top priorities in both academic and industry fields (Zellner, 2011) where it was defined by (Coskun et al., 2008) as "the most important and common titles in both literature and applications". This is explained by the fact that a process improvement has become an important task to achieve, and a crucial step in a process lifecycle. Organizations nowadays look at it as an ongoing issue that permits matching with the changing business environment by adapting business processes to technological, organizational and structural changes: it addresses the root causes of process deficiencies, analyses it and identifies areas of potential improvement in order to highlight the type of changes that could add value to the performance of a process.

For this, efforts to present effective methods/techniques for BPI were developed in literature where three holistic methods for process improvement were defined: Six Sigma, Lean Methodology and TQM (Total Quality Management). The first methodology is used to measure defects in a process and eliminates them for better quality of the final product. It consists of five phases, Define, Measure, Analyze, Improve and Control known as (DMAIC). On the opposite, Lean methodology aims at optimizing the process itself. It focuses on improving employee productivity rather than the product. While the main priority of TQM methodology is delivering value to the end-customer by focusing on the organization as a whole, rather than individual processes. 
Among the proposed BPI techniques, a model-based and integrated process improvement (MIPI) methodology was presented by (Adesola, 2005). It is a seven steps procedural approach that guides the actions and the decisions of a process design team. Another research presented by Johannsen et al., (2017) focuses on managing knowledge within the process of improvement by proposing a BPI roadmap, supporting the systematic transformation of implicit process knowledge into explicit process knowledge during the improvement process. Despite their usefulness, some of these methods were considered as the most expensive methodologies to deploy into BPI project that could negatively affect the profitability of an organization e.g. TQM, Six Sigma (Johannsen et al., 2017).

Thus, a new solution permitting better management and improvement of a BP was introduced and adopted by several BP projects. This approach called BPM (Business Process Management) is considered as "the most powerful approach for organizations to improve their performance and satisfy their customer needs" (Forster, 2006). It provides an end-to-end process understanding, visibility and control while ensuring effective communication across an organization. BPM combines BPI, performance management and organizational change management with technology to ensure the success and sustainability of process improvements.

However, it is widely agreed in literature that the most value-adding phase in a BPM project named as the act of improving, lacks sufficient guidelines and is poorly supported especially on presenting the exact instructions to improve business processes (Dumas et al., 2013; Forster, 2006). Therefore, to better represent the act of improvement of a BP, i.e. the transformation from an as-is process model to a desired to-be process model, a new era for BPI has been discovered which is the pattern concept. This concept introduces a set of solutions used for resolving a specific problem identified from a specific context. It is widely used in different fields in information systems such as Software Development (Gamma et al., 1994), Workflow Management (Van Der Aalst et al. 2003), the automation of a BP (Aldin et al., 2012) and Business Process Configuration (Weber et al. 2008; Ayora et al., 2016).

In BPI, a pattern approach is defined as an abstract form of a recurring instance of a process modification step used in a business process improvement activity (Forster, 2006) where a number of BPI approaches were defined based on this concept (Falk et al. 2013; Bergener et al. 2015; Kim et al. 2008; Yousfi et al. 2015; Nidermann et al. 2011). Despite being identified as one of the most promising approaches addressing the actual act of improvement in a process, this concept is barely used in BPI field (Falk et al., 2013; Forster, 2006). Only few works have been inspired by this concept to actually present the act of improvement phase. Also, not all of the defined approaches present a detailed description of their proposed patterns i.e. a holistic presentation of the defined patterns (attributes and their relationship). Thus, a meta-model for BPI patterns is recommended to clearly define and specify the structure of a pattern. The use of meta-models helps in establishing consistency in the description of a pattern which facilitates their application and consequently improves the performance of a process and its flexibility.

This work is part of an ongoing research study which aims to propose an approach for BPI based on a set of improvement patterns. The goal of this paper is to define a meta-model presenting the structure of a BPI pattern. It describes the core attributes of a pattern and their interrelationship. The definition of the meta-model was first based on an analysis of the literature where we highlight the research studies proposing a meta-model for patterns along with the attributes defined in it. Then, we have been based on the flexibility concept in order to represent the structure of our meta-model.

The remainder of this paper is organized as follows: section 2 gives an overview of proposed pattern meta-models in literature. In section 3, a meta-model for BPI patterns is developed, and the interrelationships between the involved attributes are shown. Afterwards, a discussion about the derived meta-model and those identified in literature is elaborated in section 4 . The conclusion in section 5 gives a summary of the presented work and deals with implications for further research.

\section{PATTERN META-MODELS IN LITERATURE}

As discussed above, this work focuses on defining a pattern meta-model for BPI. Thus, the literature review will concentrate on presenting the research studies where a meta-model for patterns was proposed as well as the approaches that have been proposed based on already defined meta-models. Before presenting the meta-model, it is necessary to distinguish between the terms "model" and "meta-model". A model is a simplified representation of a certain reality. It is based on modeling methods/tools which allow representing 
an abstraction of reality for a certain purpose. These methods are represented by a modeling language describing a (formal) notation where the elements are defined by diagrams using graphical symbols such as UML diagram, Petri Net, BPMN, etc. In contrast, a meta-model is seen as a description of another model using a modeling language. It can be defined as a model of a (modeling) language, representing an abstract syntax of all model classes of a language. To generate such meta-models, a meta-modeling process is applied. It is defined as the analysis, the development and the construction of frames, rules, constraints and models in order to model a predefined class of problems.

In fact, meta-modeling is the most desired and used concept to formalize a modeling language. It helps into using the language in a consistent way and checking for syntactic correctness of implemented rules and constraints. In the domain of BPM, many research studies use the concept of meta-modeling to represent their approach e.g. Johannsen and Fill (2017). Specifically, for BPI, several researches were inspired by the concept of meta-modeling in order to propose meta-models for patterns such as Tran et al. 2011; Falk et al. 2013 and Yousfi et al. 2015. Their proposed meta-models give an overview of the structure of a pattern by describing the different attributes used to define it and their relationships. It helps users (modelers, developers) into understanding the pattern, the way to apply it and to implement it in a specific context.

In literature, the most common descriptions of a pattern are the format proposed by Gamma et al. (1995) and that proposed by Alexander et al. (1997). They were applied in the definition of several patterns such as Business Process Patterns (Tran et al., 2011), Process Pattern (Störrle, 2001) and BPIP (Forster, 2006; Falk et al., 2013; Ayora et al., 2016; etc.). It includes the following attributes: the name of the pattern, the problem that a pattern is intended to solve, the context of applying the pattern, the solution facilitating its implementation, a pictorial representation of the pattern (image, model, architecture), the motivations that surround the use of the pattern (forces), the application of the pattern on a simplified example or a real life situation (examples) and the relationships between the defined pattern (related patterns).

Based on these elements, a number of research studies related to the definition of patterns have been defined, based on a SLR research study established in previous work (Missaoui et al., 2019). In the area of Workflow Management, Van Der Aalst et al. (2003) define a list of workflow patterns used to specify aspects related to control flows, data flows, resource assignments, etc. These patterns were defined by five attributes: Name, description, solution, related patterns and implementation. For Process Modeling domain, the work of Gschwind et al. (2008) proposes an approach of pattern application during process modeling based on the context and the consequences of applying it. The work of Cherfi et al. (2008) provides a set of quality patterns to evaluate the quality of a modeled process. The structure of the defined patterns contains the following attributes: name, problem, context, solution, related patterns and quality metrics. Tran et al. (2011) focuses on the concept of pattern reuse where they have defined an approach assuring a correct application of a given pattern and reducing modeling time. They have proposed a meta-model for process patterns where the structure is essentially presented by four attributes: name, problem, context and applied task.

In the area of BPI, a number of pattern approaches has been identified. Appleton (1997) focuses on software process improvement and provides 10 patterns for process improvement projects, described by a list of attributes such as name, context, problem, solution, related patterns, know uses, etc. Forster (2006) presents a framework defining the structure of a BPI pattern; he only describes the idea of BPIP and how to implement it without detailing its structure. Another approach proposed by Kim et al. (2008) aims at defining a set of process change patterns within BPI projects based on the structure of Workflow patterns. The defined patterns were only presented by a textual description along with a graphic representation of pattern implementation. Nidermann et al. (2011) elaborate the concept of business process optimization and propose a formalized approach for pattern optimization. The approach presents a list of optimization patterns presenting opportunities for improvement. The structure was presented in the form of an algorithm defining the specification, detection and application of the pattern. The majority of these publications simply adapt former schema of descriptions to present their patterns. As an example, the schema proposed by Gamma et al., was taken as a basis by (Störrle, 2001) to validate the proposed approach. But there are also works that emphasize the importance of describing patterns (Kim et al., 2008; Nidermann et al., 2011). However, they do not focus on presenting the structure of their patterns. Nevertheless, the work of Falk et al. (2013) is considered as "the only" research where a meta-model for BPI patterns was defined. It focuses on presenting the general structure of a BPIP by using the Design Science Research methodology (DSRM) and presenting an overview of pattern notation formats defined in literature. The meta-model includes attributes already defined in literature (name, context, problem, solution) and others related to the domain of BPI (KPI, effects). 
To sum up, despite having some recognizable pattern approaches in literature aiming to improve BPs based on the concept of patterns. There are a few works dealing with presentation of attributes that determine the structure of a pattern and providing a general and a comprehensible meta-model especially for BPI patterns. Thus, the main intention of this research is to examine and present the most important attributes to describe a BPI pattern by proposing a meta-model supporting the development of patterns as well as the creation of a pattern repository. The definition of the meta-model is based on the study of the different pattern attributes presented in literature, where we focus on those determining a BPIP and especially those presented by Falk et al. (2013) to determine those considered as necessary to define our BPIP meta-model.

\section{PROPOSED META-MODEL FOR BPI PATTERNS}

For defining the meta-model, we were based on the attributes presented in section 2. In fact, proposing an approach of BPI must consider three general aspects: Process effectiveness for better informed decision and better execution of necessary tasks; Process efficiency for improving execution time and reducing resource reuse and Process flexibility for adapting the process to any type of change affecting its performance. The last concept has been recognized as a critical quality of an effective business process that must be able to adapt to any change in the environment (Schonenberg et al. 2008). The definition of our meta-model focuses on the concept of flexibility where we have considered that the main objective of a BPI technique is to ensure greater process flexibility by permitting the process to adapt to any type of change occurring during design-time or run-time. Indeed, to qualify a process as flexible, we have to take into account four principal requirements (needs): Variability, Adaptation, Evolution and Incompleteness/Looseness (Reichert and Weber, 2008). Each of these needs may affect each of the process perspective (behavior, organization, information, time, etc.). The first type of need defines the capacity of a process to consider different variants. These variants have either the same goal or a similar one but differ depending on the context. The second presents the capacity of a process to deal with emerging events that leads to modifying the structure of a process and its execution. These events can be triggered by the appearance of several factors. The evolution need focuses on evolutionary changes that a process can have. These changes may be permanent (valid from the moment when will be implemented) or temporary (valid for a specific period of time). It represents the ability of a process to change when the corresponding BP evolves. The evolution can be triggered by external changes and/or internal changes. The common goal of these three requirements is to ensure the flexibility of the process by taking into consideration the types of changes and their context. However, for the incompleteness need we generally focus on loosely defined models, i.e. in this type, only the objectives of the process are known in advance which results in a not fully defined process model. In this case, the design phase must be completed during the execution phase where the changes concern only one part of process definition. Based on these requirements and taking into consideration the work of (Falk et al., 2013) as well as the pattern format of (Gamma et al. 1995), we have developed our own meta-model for BPI pattern which corresponds to a graphical description of a BPI pattern highlighting its core attributes and presenting four types of BPIP: variability pattern, adaptation pattern, evolution pattern and looseness pattern. These patterns were derived from the defined requirements where we have considered that an improved and a flexible process adopt one (or more) of these patterns, by being based on the context to which it will be applied.

The meta-model, as described in Figure 1, details the structure of a BPI pattern presenting the core attributes of a pattern (context, problem, solution) and other attributes related to its definition. In the center of the model, the class presents the BPI pattern which has a name as its identifier and a set of methods (add, remove, modify, select) on which the process of pattern definition and selection will be based on. Every pattern is specified with a single problem to solve, a single solution to present and a single context to address. The context attribute is defined based on a set of drivers, facilitating the definition and the classification of patterns. The problem concerns a specific BP lifecycle phase described by its characteristics and a description of the corresponding phase. The solution presents a number of mechanisms, defining the way to apply a pattern into a set of case studies. The implementation depends on the purpose of the case study and its effect on time, cost, quality and flexibility values. The meta-model presents four types of patterns: Variability patterns, Adaptation patterns, Evolution patterns and Looseness patterns. Each type possesses its own meta-model, defined for a specific problem and a specific context where each meta-model includes the general attributes of BPIP and others specific to the defined type. 


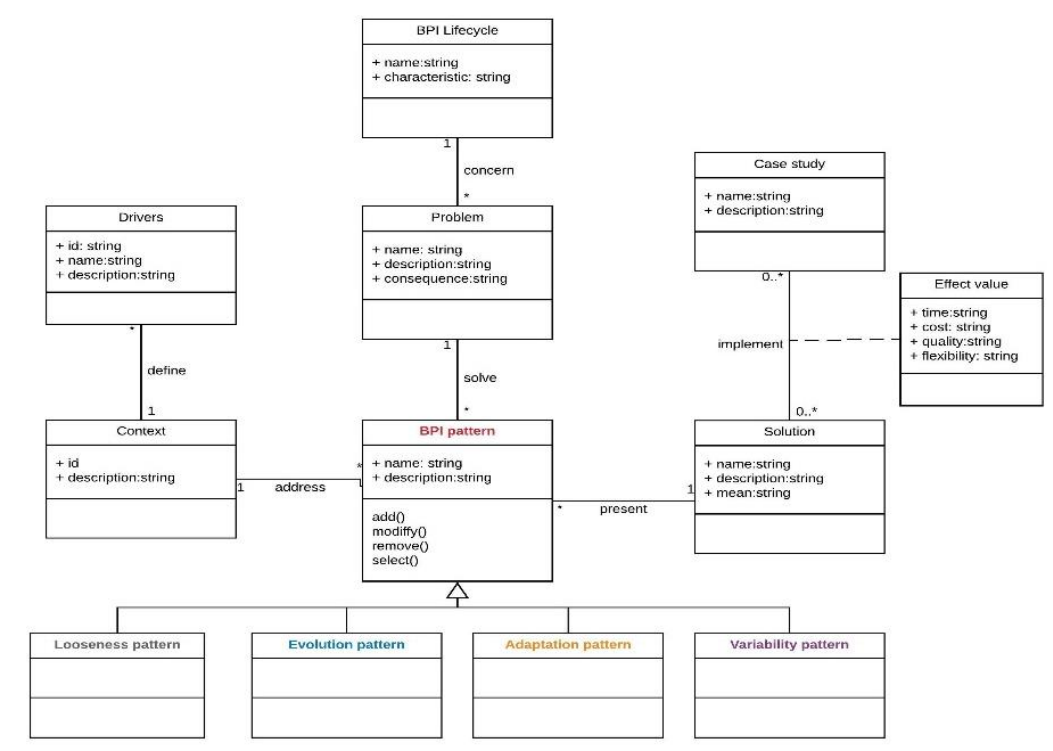

Figure 1. Meta-model for business process improvement pattern

\subsection{A Meta-Model for Variability Patterns}

The first type is variability pattern (see Figure 2 left picture) used to model and execute a variable business process. It is presented by a specific solution and concerns a specific variability objective describing the conflicts handled by a variable BP, related to a specific process family. A variable BP can have one to several variable process parts describing the object of change. These parts can be associated to different variant points defining the subject of changes which is the place where the variation occurs: variants can be removed or included from variant points by changing the flow of events, the sequence flow or the activities of the model. These changes are applied by a number of variability mechanisms that permit adding, removing, modifying or replacing a variant.

\subsection{A Meta-Model for Adaptation Patterns}

The second type of BPIP is the adaptation pattern. It allows a structured modification of a process schema by using a number of operations. The structure of the pattern is presented by the core attributes of BPIP and those specific to the presented pattern. As presented in Figure 2 (right figure), the adaptation pattern is based on a number of adaptation changes determined by the framework of application and the type of adopted changes (high level changes, small changes). These adaptations are triggered by a specific factor that can be internal or external and applied into several process parts using a number of operations. These operations can be applied at high level by adding new activities or an entire process fragment or at low-level by adding or deleting a single node.

\subsection{A Meta-Model for Evolution Patterns}

The evolution pattern considers the evolutionary changes that a process can have. It is triggered by a specific type of drivers defined by the context attribute. These drivers have two types: internal drivers (design errors, technical problems, and poor model quality) and external drivers. It is presented by a specific solution that is based on a number of evolution changes (see Figure 3 on the left) defined in a category of evolution presenting the concepts of incremental evolution, continuous evolution or revolutionary evolution. These evolutions are introduced by a set of operators determining the way to add, remove, modify one to several process artefacts. These artefacts comprise information about the structure of the process and define the element(s) on which the pattern is being applied on (role, activity, event, sub-process, etc.). 


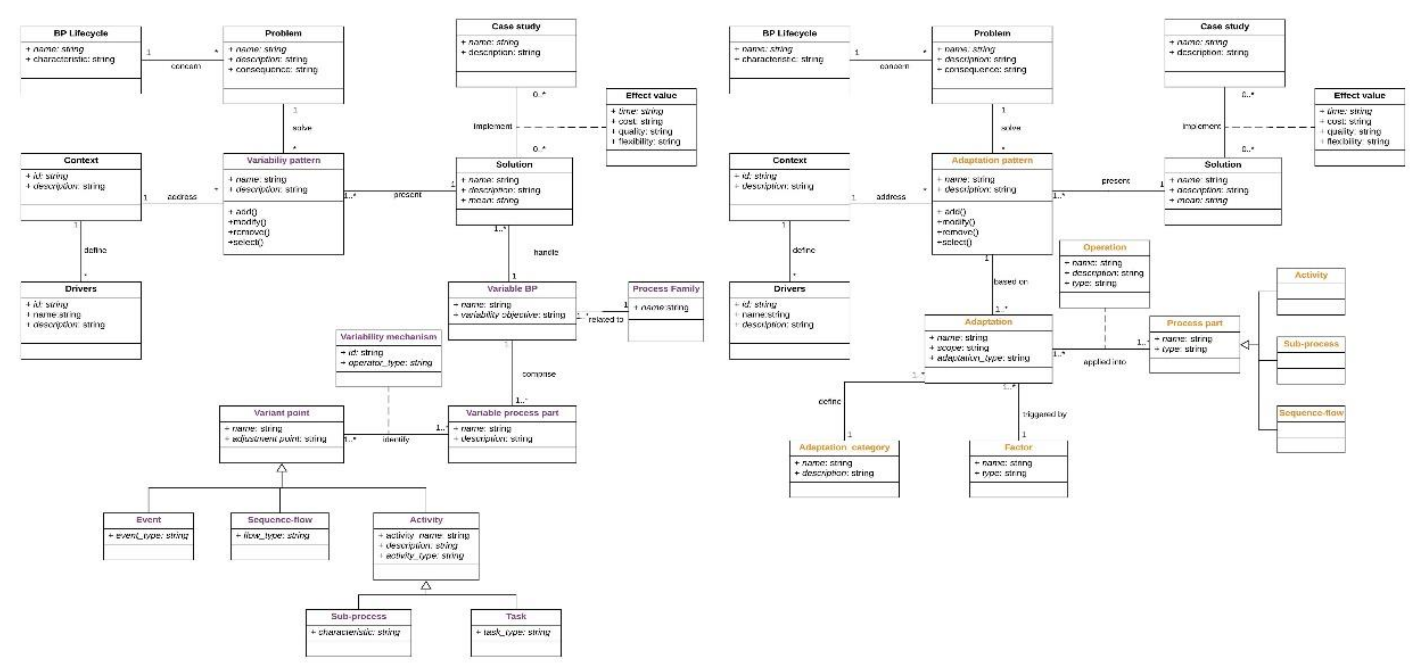

Figure 2. Meta-models for Variability pattern (left) and adaptation pattern (right)

\subsection{A Meta-Model for Looseness Patterns}

This type of pattern focuses on loosely specified models where only their goal is known in advance and the parameters determining the exact cause of an action are typically not known and might change during process execution. To deal with this, decisions regarding the exact specification of selected parts of the process have to be defined. These decisions can be defined, as demonstrated in Figure 3 (on the right), by a loosely point presenting the loosely specified parts: it can concern the control flow part in a BP or the activity part. These points are presented by a category of loosely points defining different types of looseness and expressed by a number of compliance rules determining the exact type of action to apply on the part. The definition of these rules is based on a number of constraints and a set of analysis techniques that present a number of metrics allowing to measure the degree to which these rules have been respected during the modeling of a single loosely point.

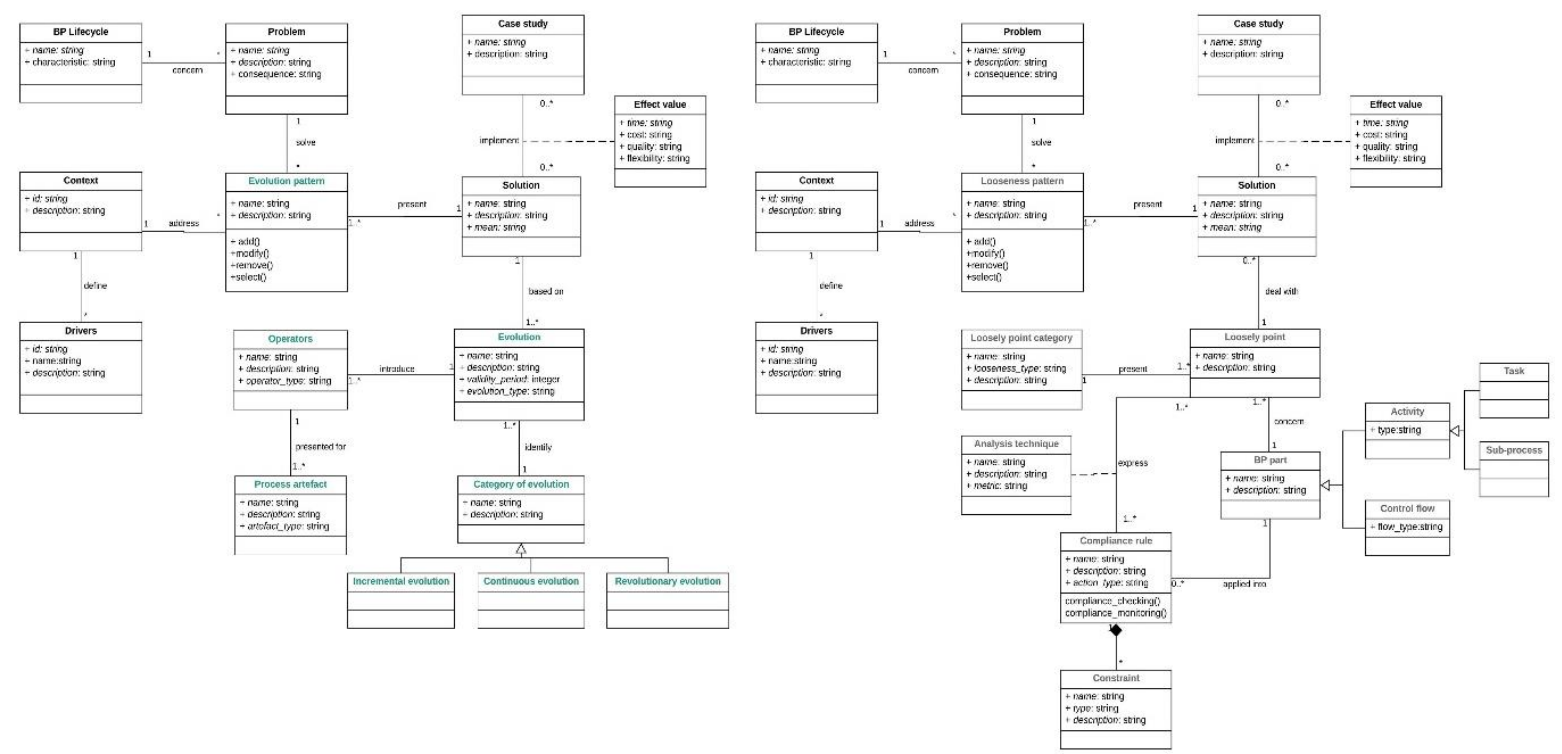

Figure 3. Meta-models for evolution (left) and looseness patterns (right) 


\section{DISCUSSION}

This paper aims to proposing a meta-model for BPI patterns, presenting the general structure of an improvement pattern based on the flexibility concept. It includes four types: Variability, adaptation, evolution and Looseness patterns where each type has its own model. In fact, in literature, all of the defined meta-models focus only on one flexibility need where we may cite the work of (Yousfi et al., 2015) for variability needs, (Nidermann et al., 2011; Weber et al., 2008) for adaptation need.

For evolution need we have the works of (Forster, 2006; Falk et al., 2013) and Reichert and Weber, (2012) for Looseness need. This means that each of the proposed patterns can be applied only in the context in which it was defined. Among these studies, we found that only the work of (Falk et al., 2013) proposes a meta-model for BPIP while focusing on the evolution needs of a process. So overall, we can conclude that so far no work has proposed a complete meta-model for BPIP that includes all of these needs. Thus after reviewing the literature and determining its shortcomings, we defined a meta-model presenting the global structure of an improvement pattern. The proposed meta-model may serve as a reference for defining any type of BPI pattern by only determining the context to which it will be applied. Also, it can be considered as a tool for pattern selection and categorization since it provides a global overview of BPIP structure which will facilitate the process of pattern definition and use. As with every research, our work is subject to limitations. A first one concerns the completeness of the meta-model as we covered only one concept of BPI which is process flexibility. We can justify this by arguing that a flexible BP can be easily adapted to any type of change which will reduce modeling as well as execution time of a process, eliminate waste time and permit better quality of a BP. As a consequence, the proposed meta-model set intends to be complete in the sense that it allows the definition of an improvement pattern, covering all possible improvement aspects. Also, the work lacks a validation of the defined meta-model where we are in the process of implementing the defined structure on a real process describing the workflow of patient treatment within the emergency department.

\section{CONCLUSION}

We proposed a meta-model describing the structure of BPI patterns based on process flexibility. It presents a number of attributes considered as essential to the definition of a BPIP. Our work is, to the best of our knowledge, the only one which presents a complete structure of a BPI pattern by regrouping all BPIP types into a single meta-model that determines the core attributes of any improvement pattern as well as attributes specific to the type of the pattern. This will certainly facilitate the process of pattern selection and application. However, we cannot claim the feasibility of the meta-model, considering that no evaluation step was introduced. For this, the evaluation will take place in further research studies as we plan to define a list of BPIP based on the meta-model and demonstrate the way to apply it on real example of BP models. The definition of these patterns will essentially be based on the context attribute from which we will be able to categorize these patterns. Then, we will develop a prototype based on a pattern database that we will create and conduct experiments to study the impacts of these patterns on the BP lifecycle. This will help us in validating the feasibility of our patterns and presenting an approach for pattern definition and reuse.

\section{REFERENCES}

Adesola, S. and Baines, T., 2005. Developing and evaluating a methodology for business process improvement. In BPM Journal, Vol. 11, No.1, pp. 37-46.

Aldin, L., Alfaris, A. and De Cesare, S. 2012, Business process pattern automation: State of the art and research challenges. IADIS International Conference Information Systems. Berlin, Germany, pp. 175-185

Alexander, C., Ishikawa, S., Silverstein, M., Jacobson, M., Fiksdahl-King, I., and Angel, S., 1997. A pattern language. Towns, Buildings, Construction. Oxford University Press, New York.

Appleton, B., 1997. Patterns for conducting process improvement. Conference on Pattern Languages of Program Design.

Ayora, C., Torres, V., De la Vara, J.L. and Pelechano, V., 2016. Variability management in process families through change patterns. In Journal of Information and Software Technology, Vol. 74, pp. 86-104. 
Bergener P, Delfmann P, Weiss B and Winkelmann, A., 2015. Detecting potential weaknesses in business processes: an exploration of semantic pattern matching in process models. In BPM Journal, Vol. 21, No. 1, pp. 25-54.

Cherfi, S.S, Comyn-Wattiau, I. and Akoka, J., 2008. Quality patterns for conceptual modeling. International Conference on Conceptual Modeling-ER.. Springer, Heidelberg, pp. 142-153.

Coskun, S., Basligil, H., and Baracli, H., 2008. A weakness determination and analysis model for business process improvement. In BPM Journal, Vol.14, No.2, pp. 243-261.

Davenport, T., 1993. Process Innovation-Reengineering work through Information Technology. Harvard Business School Press, Boston.

Dumas, M., Rosa, M.L., Mendling, J. and Reijers, H., 2013. Fundamentals of Business Process Management, Springer, Berlin, Heidelberg.

Falk, T., Griesberger, P. and Leist, S., 2013. Patterns as an artifact for business process improvement -insights from a case study. International Conference on Design Science Research in Information Systems and Technology. Helsinki, Finland, pp. 88-104.

Forster, F., 2006. The Idea Behind Business Process Improvement: Toward a Business Process Improvement Pattern Framework. Journal of BPTrends, pp. 1-14.

Gamma, E., Helm, R., Johnson, R. and Vlissides, J., 1994. Design Patterns: Elements of Reusable Object-Oriented Software. Addison-Wesley Professional, Upper Saddle River, United States.

Gschwind, T., Koehler, J. and Wong, J., 2008. Applying patterns during business process modeling. The 6th International Conference on Business Process Management. Springer, Heidelberg, Vol. 5240, pp. 4-19.

Johannsen, F., and Fill, H.G., 2017. Meta Modeling for Business Process Improvement. In Journal of Business \& Information Systems Engineering, Vol. 59, No. 4, pp.251-275.

Kim, D., Kim, M. and Kim, H., 2007. Dynamic business process management based on process change patterns. International Conference on Convergence Information Technology. Gyeongju, South Korea, pp. 1154-1161.

Missaoui N. and Ayachi Ghannouchi S., 2019. Pattern-Based Approaches for Business Process Improvement: A Literature Review. Park J., Shen H., Sung Y., Tian H. (eds) Parallel and Distributed Computing, Applications and Technologies. PDCAT 2018. Communications in Computer and Information Science, Springer, Singapore. Vol. 931, pp.390-400.

Nidermann, F., Radeschütz, S. and Mitschang, B., 2011. Business process optimization using formalized optimization patterns. International conference on Business Information System. Springer, Heidelberg, pp.123-135.

Reichert, M. and Weber, B., 2012. Enabling flexibility in Process-Aware Information Systems: Challenges, Methods, and Technologie. Springer, Verlag, Berlin, Heidelberg.

Rinderle-Ma, S., Reichert, M. and Weber, B., 2008. On the formal semantics of change patterns in process-aware information systems. International conference on conceptual modeling-ER. Springer, Heidelberg, pp. 279-293.

Schonenberg, H., Mans, R., Russell, N., Mulyar, N. and Van Der Aalst, W., 2008. Process flexibility: a survey of contemporary approaches: Advances in Enterprise Engineering. Springer-Verlag, Berlin, pp. 16-30.

Störrle, H., 2001. Describing process patterns with UML. Europian Workshop on Software Process Technology, Software Process Technology. Springer, Heidelberg, Vol. 2077, pp.173-181.

Tran, H.N., Coulette, B., Tran, D.T. and Vu, M.H., 2011. Automatic reuse of process patterns in process modeling. Proceedings of the ACM Symposium on Applied Computing TaiChung. Taiwan, NY, USA, pp. 1431-1438.

Van Der Aalst, W.M.P., Ter Hofstede, A.H.M., Kiepuszewski, B. and Barros, A.P., 2003. Workflow Patterns. In Journal of Distributed and Parallel Databases, Vol. 14, No.3, pp.5-51.

Weber, B., Reichert, M. and Rinderle Ma, S., 2008. Change patterns and change support features- enhancing flexibility in process-aware information systems. In Journal of Data \& Knowledge Engineering, Vol. 66, No.3, pp. 438- 466.

Yousfi, A., Saidi, R. and Dey, A.K., 2016. Variability patterns for business processes in BPMN. In Journal of Information Systems and e-Business Management, Vol.14, No.3, pp.443-467.

Zellner, G., 2011. A structured evaluation of business process improvement approaches. In BPM Journal, Vol. 17, No.2, pp.203-237. 